\title{
High Resolution Observations of Young Stellar Objects with Extreme-AO Systems: Jets, Disks, and Accreting Companions
}

\author{
Simone Antoniucci* \\ INAF - Osservatorio Astronomico di Roma \\ E-mail: simone.antoniucci@inaf.it
}

\begin{abstract}
State-of-the-art high-contrast optical and near infrared instruments equipped with Extreme Adaptive Optics (ExAO) systems have recently started to provide high-resolution images of Young Stellar Objects (YSOs) of unprecedented quality, which are revolutionising our view and understanding of the structures around young sources. Narrow-band ExAO observations of jets from YSOs (the first ones of which were carried out with VLT/SPHERE) are able to resolve for the first time the base of the jets and provide crucial clues to discriminate the jet launching region and mechanism, which is key to evaluate the jet feedback on the disk and eventually on the structure of planetary systems. High-contrast observations of protoplanetary disks have been hugely successful in providing fundamental insights on the disk structures (spiral arms, gaps, rings). These results, in conjunction with ALMA data, have fostered a great advancement in the development of disk evolution models, which take into account gas and dust interaction with forming planets within the disk. Most observations with ExAO instruments have focused in particular on the detection of these young planets. A very promising technique to detect actively accreting giant planets still in formation is based on narrow-band imaging in accretion tracers such as $\mathrm{H} \alpha$. Upcoming ExAO instruments like SHARK-VIS at LBT will be specifically optimised to perform these kind of observations in $\mathrm{H} \alpha$ on large samples of YSOs. The final aim is to provide constraints on giant planet formation models and to asses the effects of planetary formation on the evolution of the observed disk structures.
\end{abstract}

Accretion Processes in Cosmic Sources - II - APCS2018

3-8 September 2018

Saint Petersburg, Russian Federation

\footnotetext{
* Speaker.
} 


\section{Extreme AO systems}

The latest years have seen a great advancement in the study of disks around Young Stellar Objects (YSO) in particular in relation with planet formation. This advancement has been made possible not only by the unique data provided by ALMA at millimetre wavelengths but also by the (quasi) diffraction-limit complementary observations at optical and near-infrared (NIR) wavelengths provided by last-generation high-angular-resolution and high-contrast instruments on $8 \mathrm{~m}$ class telescopes equipped with Extreme Adaptive Optics (ExAO) systems.

ExAO systems (e.g. Guyon 2018) are Adaptive Optics systems optimized to deliver highorder correction of the wavefront corrupted by the atmospheric turbulence, using a relatively bright natural guide stars. They generally have faster and improved wavefront sensing capabilities than standard AO systems, employ more actuators, and multiple deformable mirrors. The final aim of these facilities, mostly driven by the needs of current exoplanet search programmes, is producing images close to the diffraction limit while achieving the best possible contrast at small angular separation from the bright guide star. These images are usually processed with ad-hoc post-facto techniques aimed at improving the image contrast, based on various types of differential imaging, such as, e.g., Angular Differential Imaging (ADI), Spectral Differential Imaging (SDI), or PDI (Polarimetric Differential Imaging). Most of the biggest telescopes in the world are now equipped with ExAO systems that feed optical/NIR instruments: the Large Binocular Telescope (LBT) with LUCI and the upcoming SHARKs, SUBARU with SCExAO, GEMINI with GPI, Magellan with MagAO, and the Very Large Telescope (VLT) with SPHERE. On 8m telescopes, images obtained with such instruments approach angular resolutions $(\lambda / \mathrm{D})$ of the order of 30 mas at $1 \mu \mathrm{m}$ (corresponding to 4au at $150 \mathrm{pc}$ ) and 15 mas at $0.5 \mu \mathrm{m}$ ( $2 \mathrm{au}$ at $150 \mathrm{pc}$ ), with contrasts that can be as low as $10^{-6}$ at separations larger than 200-300 mas from the central star. By providing this unique combination of high angular resolution and high contrast, ExAO instruments are now allowing the community to study the faint structures and companions around nearby YSOs and to shed light on their the morphology, dynamics, and evolution.

In the following sections, I will briefly outline some of the results recently obtained in the field of YSOs with new ExAO instrumentation, focusing in particular on VLT/SPHERE imaging of jets (Sect. 1) and on observations of disks and forming (i.e. still accreting) companions (Sect. 2).

\section{Observations of YSO jets: revealing the launching mechanism}

In the standard paradigm for the formation of solar type stars, ejection of matter in form of winds and collimated jets is tightly connected to the mass accretion process from the circumstellar disk (e.g. Frank et al. 2014). Jets are indeed an essential feature of the process of star formation, since they are believed to play an important dynamical role as one of the main agents that can remove the angular momentum from the circumstellar disk, thus allowing matter to accrete onto the central star. While it is now widely accepted that the combined action of magnetic fields and rotation is required to explain the efficient jet collimation and acceleration (Cabrit 2007), several MHD ejection sites may operate in parallel: the stellar surface, the inner disk edge (X-winds), or a broader range of disk radii (disk winds). Their relative contribution to the jet remains subject to debate (Ferreira et al. 2006 and references therein). This is due to the fact that because of the 
spatial scales involved (a few au) and of the high contrast between jet and star, the jet-launching region has remained basically unexplored up to now from an observational point of view. In the best cases, using the Hubble Space Telescope and AO-assisted ground-based instruments, it was possible to trace the jet down to a distance of 0.1 arcsec from the source (e.g. Agra-Amboage 2014, Maurri et al. 2014), corresponding to about 15 au for nearest young objects.

Identifying the size of the jet launching region would be crucial tu establish the jet efficiency in removing the angular momentum from the system and to understand how jets affect the disk evolution and planetary formation. For instance, if jets are emitted from an extended region of the disk, as expected in MHD disk-winds, the structure of the accretion disk and its surface density would be remarkably different with respect to standard models of viscous disks (Combet \& Ferreira 2008). In addition, the magnetic braking in the region of the jet launch, which extends up to 10-15 au from the star for a classical disk wind, could have an important influence on the aggregation of material and the formation of planetesimals.

In order to gain insight into the mechanism of jet acceleration and on its feedback on the disk it is however fundamental to observe the base of the jet as close as possible to the source. This basically means breaking previous observational limits and probe the region within $\sim 5-15$ au (i.e. $0.04-0.1$ arcsec for nearby star-forming regions), where the jet has not been significantly perturbed yet by the interaction with the ambient medium and where the signatures of the launching mechanism are still imprinted in the flow morphology.

New high-contrast instruments with ExAO correction in the optical/NIR can now break this limit for the first time and provide unprecedented observations of the base of the jets. In particular, observations at scales below the $0 " .1$ aim at investigating the width of the jet at its base, which is an important quantity to discriminate the different launching regions and test model predictions on the jet collimation. Achieving a good sampling of the jet width on spatial scales $<50$ au would allow for instance to estimate the jet width at launch by interpolation (the so-called jet footprint), so as to derive the size of the region where the jet originate. Another goal of the observations is to study the global morphology of the jet stream close to the star. For instance, the distribution of jet knots close to the source can provide indication on a pulsed accretion with related bursts of mass ejection and would allow to determine their typical time-scales. Analogously, the detection of a wiggling of the jet on short spatial-scales might indicate the presence of unseen close-in companions (e.g. Antoniucci et al. 2016) or testify an active precession process.

\subsection{First YSO jet observations: SPHERE on Z CMa}

The first demonstration of the potential of ExAO-assisted observations of jets was provided by Antoniucci et al. (2016), who used SPHERE/ZIMPOL (Schmid et al. 2018) at the VLT to target the young binary Z CMa. This is a noteworthy system (e.g. Whelan et al. 2010, Bonnefoy et al. 2017) in which both components - an intermediate-mass Herbig Be star with recurrent outbursts and a FUor-type object (e.g. Audard et al. 2014) - drive a jet. Although its fairly large distance $(\mathrm{d}=1150 \mathrm{pc})$ prevented to explore spatial scales below $10 \mathrm{au}$, this target offered the opportunity to image two jets at the same time from peculiar outbursting objects.

As jets emit in atomic and molecular lines (which originate in the radiative cooling zones of shocks between the ejected matter and the ambient medium), the authors performed narrow-band imaging observations using two typical optical jet tracers: the [OI] at $6300 \AA$ and $\mathrm{H} \alpha$. To improve 

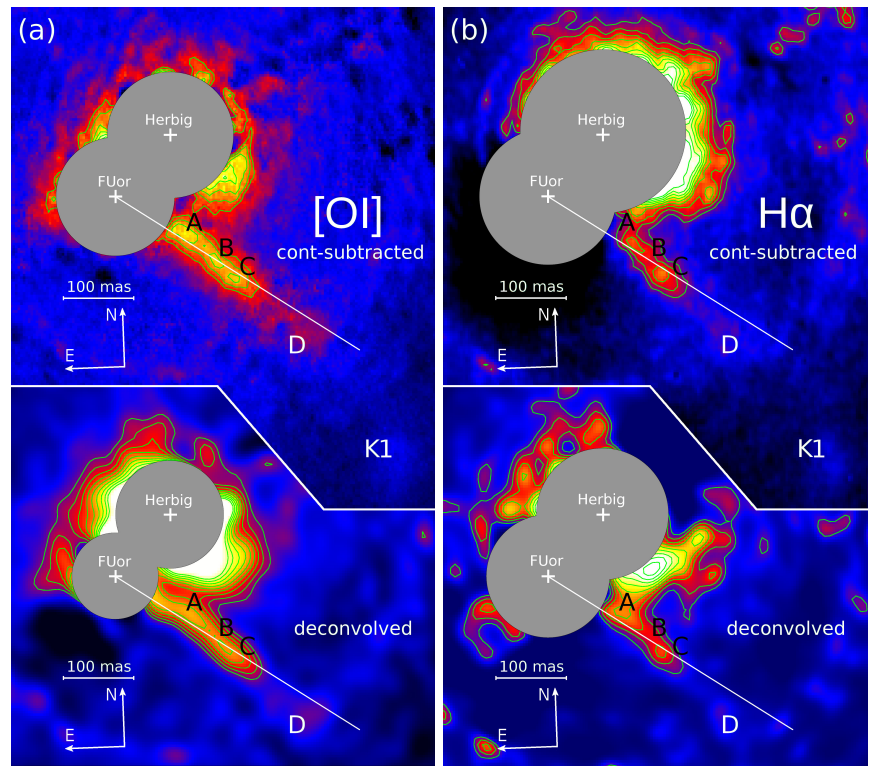

(c)

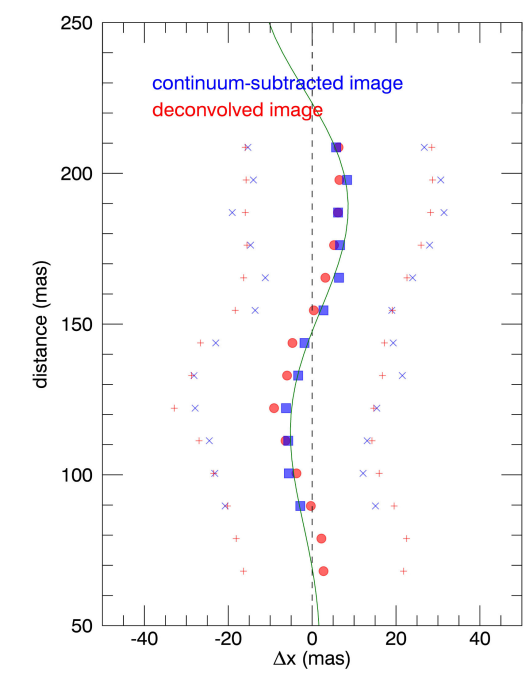

Figure 1: ZIMPOL images of the Z CMa system jets, adapted from Antoniucci et al. (2016). (a):[OI] continuum-subtracted (top) and deconvolved (bottom) images; (b): same for the H $\alpha$ observations. The centroids of the two stars and the position of the main knots of the FUor jet are indicated, while areas heavily corrupted by artefacts around the star centroids have been masked. (c): Peak and widths of the FUor jet spatial profile as a function of the distance from the source for both the [OI] continuum-subtracted (blue squares) and deconvolved images (red circles). Blue crosses and red pluses mark the measured profile width the two images. The solid green line is the best-fit of the wiggle produced by an orbital motion of the jet source around a companion, obtained following the model by Anglada et al. (2007).

the contrast at small separations from the driving source, images with a narrow-band filter on the adjacent continuum were also acquired to subtract the stellar continuum (SDI technique).

The continuum-subtracted OI and $\mathrm{H} \alpha$ images of $\mathrm{Z} \mathrm{CMa}$ are shown in the upper part of Fig. 1a,b, while deconvolved images (showing consistent structures) are displayed in the lower part. The ZIMPOL images reveal extended emission from both sources: a compact and poorly collimated wind emission SW of the Herbig star and a more extended and collimated jet from the FUor component. The authors were able to analyse in detail the FUor jet, by considering contiguous image slices orthogonal to jet axis, so as to obtain the spatial profiles of the jet at various distances. By fitting Gaussians to these profiles, the peak position and width as a function of the distance from the star were obtained, which are reported in the schematic shown in Fig. 1c for both the continuum-subtracted and deconvolved [OI] image. The FUor jet is traced down to about 70 mas from the source ( 80 au at the distance of the target) and shows a width of 26-48 au at distances 100-200 au. This testifies a collimation similar to that observed in jets from T Tauri stars, which is a strong indication that the same magneto-centrifugal jet-launching mechanism is also operating in FUors. The displacement of the peak position clearly reveals a jet wiggling that, considering estimates of the jet proper motion (Whelan et al. 2010, Antoniucci et al. 2016) occurs on time-scales of a few years.

Based on these very short time-scales, this wiggling can be interpreted as due to the reflex motion of the jet driving source around a very close companion. Using the formulation by Anglada 

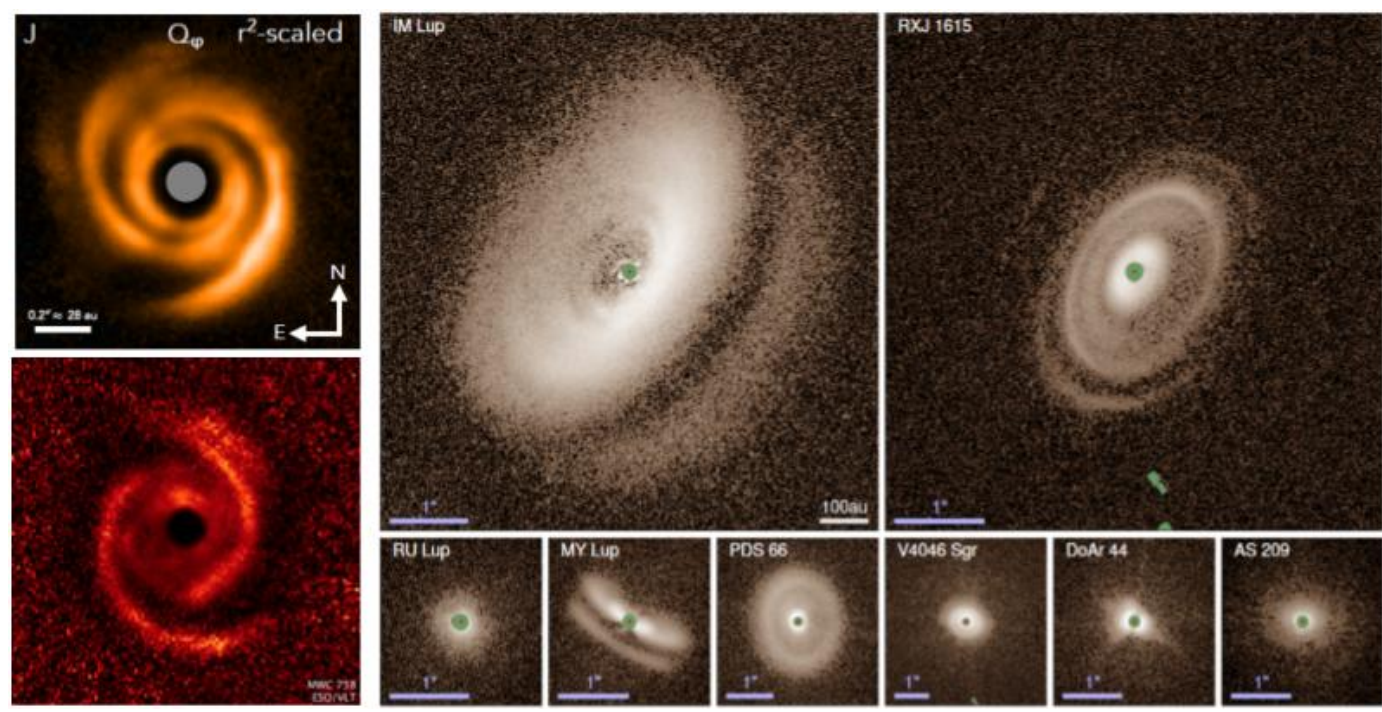

Figure 2: Example of NIR images of protoplanetary disks recently obtained with SPHERE/IRDIS on VLT. Left top: polarized scattered light images of HD 135344B in the J band by Stolker et al. (2017); left bottom: MWC 758 as observed by Benisty et al. 2016; right: several disks around T Tauri sources by Avenhaus et al. 2018 (right).

et al. (2007), Antoniucci et al. (2016) estimated an orbital motion with a period of $4.2 \mathrm{yr}$ around an unseen companion at separation 2.6-3.8 au and with mass between 0.48 and $1 \mathrm{M}_{\odot}$.

Finally, the ZIMPOL images also allowed to infer the first direct $\dot{M}_{\text {jet }}$ measurement from a jet in a FUor, using the [OI] jet luminosity. The derived jet mass loss rate $\left(\dot{M}_{\text {jet }}=1 \times 10^{-8}-1 \times 10^{-6}\right.$ $\left.\mathrm{M}_{\odot} \mathrm{yr}^{-1}\right)$ when compared to previous mass accretion rate estimates $\left(\dot{M}_{a c c}=7.9 \times 10^{-5} \mathrm{M}_{\odot} \mathrm{yr}^{-1}\right.$, Hartmann \& Kenyon 1996) would imply a very low mass-ejection efficiency $\left(\dot{M}_{j e t} / \dot{M}_{a c c} \lesssim 0.02\right)$, lower than the one typical of $\mathrm{T}$ Tauri stars.

\subsection{Upcoming new results from SPHERE}

New observations with SPHERE on VLT have been recently carried out within the framework of SPHERE GTO programme devoted to YSO jets. In particular, images of the Classical T Tauri star DG Tau have been obtain with ZIMPOL in $\mathrm{H} \alpha$ and [OI] (Antoniucci et al., in prep.), in which the jet is traced down to 40-60 mas from the source (corresponding to 5-7 au). These are probably the highest resolution images ever taken of a jet from a YSO and provide an unprecedented view of the base of the jet.

In addition, SPHERE observations of jets from the sources RY Tau and HD 163296 (both in the optical and in the near infrared) are currently being analysed in a work (Garufi et al., in preparation) aimed at investigating the connection between the observed properties of the jet and the features of the disk, which have been revealed by complementary SPHERE and ALMA observations of the targets.

\section{Disks and forming planets}

Optical/NIR high-contrast imagers have been extremely successful in providing images in 

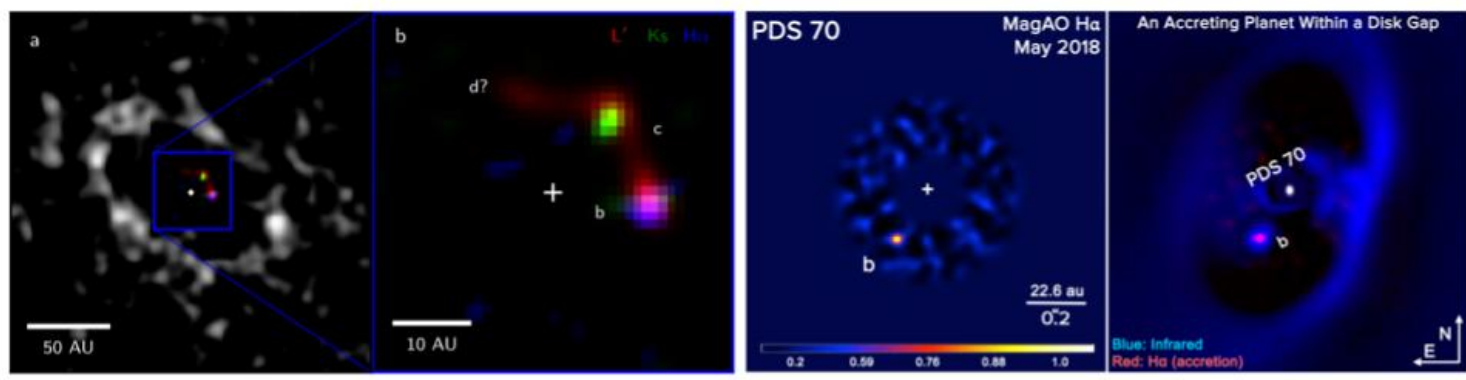

Figure 3: First reported detections of $\mathrm{H} \alpha$ emission from planetary companions, which is an indication of active ongoing accretion on these object. Left: (adapted from Sallum et al. 2015) LkCa15 b detection in $\mathrm{H} \alpha$ (in blue) is superposed to images obtained in the $\mathrm{L}$ and $\mathrm{K}$ bands (red and green). Right: (adapted from Wagner et al. 2018) PDS70 b detection in $\mathrm{H} \alpha$ shown using a red-color scale, is superposed to continuum emission in the NIR, where the inner disk cavity can be easily observed. Both results were obtained using the ExAO MagAO instrument mounted on the Magellan telescope.

scattered light of protoplanetary and debris disks around YSOs, which have revealed a great deal of details, such as condensations, rings, gaps, shadows, and spiral arms (see Fig. 2 and, e.g., Avenhaus et al. 2018, Stolker et al. 2017, Garufi et al. 2016, Benisty et al. 2016, Perrot et al. 2016, D'Orazi et al. 2018). These images, together with their millimetre counterparts provided by ALMA (e.g. Andrews et al. 2016, Cieza et al. 2017, Isella et al. 2018, Fedele et al. 2018, Kataoka et al. 2016), have totally revolutionised our vision of YSO disks and fostered an unprecedented development of sophisticated models that describe the disk structure and evolution, taking into account the interaction between gas and dust and the planets forming within the disk itself (e.g. Dipierro et al. 2015, 2018, Dong et al. 2015, 2016, 2018). In particular, many of the gaps in the dust distribution observed in protoplanetary disks might be attributed to the action of forming planets that clear the disk along their orbit.

On the basis of the scenario where most of the structures observed in disks are indeed induced by forming (giant) planets, many observations with ExAO instruments have focused on the direct detection of such planets. Although their detection is extremely challenging (expected contrasts are typically below $10^{-5}$ ), direct imaging is the only technique sensitive to giant planets in wide orbits (typically $>5 \mathrm{au}$ ). During the early phases of their evolution (the first few million years), giant planets are hot, so that they can be revealed through thermal emission (at T>2000 K) in the NIR, and may be still be accreting gas, so that they are detectable using accretion line tracers such as $\mathrm{H} \alpha$ at optical wavelengths.

In particular, the relation between $\mathrm{H} \alpha$ emission and accretion rates has been first examined by Close et al. (2014), who showed that the contrast at $\mathrm{H} \alpha$ may be much more favorable than at other wavelengths for accreting low-mass and planetary companions. This was confirmed by HST observations of GQ Lup b and other very low-mass objects with masses in the brown dwarf/planet transition regime by Zhou et al. (2014), with fairly high contrasts in $\mathrm{H} \alpha$ of the order of $10^{-3}$.

In such cases, the measured $\mathrm{H} \alpha$ flux can also be used to derive an estimate of the mass accretion rate of the planets, using empirical relationships calibrated on low-mass $\mathrm{T}$ Tauri objects and under the assumption of magnetospheric-like accretion (e.g. Alcalá et al. 2017). Gas giant planets are believed to form in circumstellar disks mainly by two distinct mechanisms, i.e. via accretion 
of gas onto rocky/icy cores of a few earth masses (e.g. Pollack et al. 1996) or by gravitational instabilities in the circumstellar disk (e.g. Boss 2001). As these models generally predict different time-scales and rates for the formation of the companions (e.g. Bowler et al. 2011, Stamatellos \& Herczeg 2015), H $\alpha$ observations can effectively provide fundamental constraints to discriminate between the two formation channels. On these bases, the detection of accreting giant planets through $\mathrm{H} \alpha$ emission represents one of the most exciting challenges for ExAO observations of YSOs.

An interesting and ideal class of targets in this respect is represented by sources with transitional disks, i.e. objects characterized by inner cavities of several astronomical units in their dust distribution (e.g. Espaillat 2014), presumably created by forming planets, which however show indication of significant amounts of residual circumstellar gas in the cavity (e.g. Manara et al. 2014). Not surprisingly, the first claimed detections of actively accreting planets observed in $\mathrm{H} \alpha$ have been reported for transitional disk sources (see Fig. 3). First, Sallum et al. (2015) have observed H $\alpha$ emission with MagAO from a candidate planetary companion in the gap of the disk around LkCa 15, although this detection has been recently questioned (Mendigutia et al. 2018). More recently, Wagner et al. (2018) have detected with MagAO the $\mathrm{H} \alpha$ emission from PDS70 b, which provides a clear indication of ongoing accretion onto the planet. This latter had been discovered just a few weeks before within the inner cavity of the PDS70 disk using SPHERE/IRDIS NIR observations (Müller et al. 2018, Keller et al. 2018).

Albeit observations at optical wavelengths are more challenging than in the NIR because of the less efficient correction provided by the AO systems, these first detections show the great potential of the $\mathrm{H} \alpha$ imaging to probe the formation process of giant planets. In this framework, the upcoming ExAO instrument SHARK-VIS at LBT (e.g. Pedichini et al. 2017, Li Causi et al. 2017, Mattioli et al. 2018), which is currently being built at the INAF-Osservatorio Astronomico di Roma and will see its first light at the end of 2019, has been designed and specifically optimized to perform SDI observations in $\mathrm{H} \alpha$ to search for accreting companions. SHARK-VIS will perform a survey on a large sample of T Tauri stars (e.g. in Taurus- Auriga) in synergy with its companion NIR instrument SHARK-NIR (e.g. Farinato et al. 2018, which is built at the INAF-Osservatorio Astronomico di Padova and optimized to detect thermal emission from young planets), thus providing crucial results on the formation mechanism of giant planets and further assessing their role in the disk evolution.

\section{References}

[Agra-Amboage et al.(2014)] Agra-Amboage, V., Cabrit, S., Dougados, C., et al. 2014, A\&A, 564, A11

[Alcalá et al.(2017)] Alcalá, J. M., Manara, C. F., Natta, A., et al. 2017, A\&A, 600, A20

[Andrews et al.(2016)] Andrews, S. M., Wilner, D. J., Zhu, Z., et al. 2016, ApJ1, 820, L40

[Anglada et al.(2007)] Anglada, G., López, R., Estalella, R., et al. 2007, AJ, 133, 2799

[Antoniucci et al.(2016)] Antoniucci, S., Podio, L., Nisini, B., et al. 2016, A\&A, 593, L13

[Audard et al.(2014)] Audard, M., Ábrahám, P., Dunham, M. M., et al. 2014, Protostars and Planets VI, 387

[Avenhaus et al.(2018)] Avenhaus, H., Quanz, S. P., Garufi, A., et al. 2018, ApJ, 863, 44 
[Benisty et al.(2015)] Benisty, M., Juhasz, A., Boccaletti, A., et al. 2015, A\&A, 578, L6

[Benisty et al.(2017)] Benisty, M., Stolker, T., Pohl, A., et al. 2017, A\&A, 597, A42

[Bonnefoy et al.(2017)] Bonnefoy, M., Chauvin, G., Dougados, C., et al. 2017, A\&A, 597, A91

[Boss(2001)] Boss, A. P. 2001, ApJ, 563, 367

[Bowler et al.(2011)] Bowler, B. P., Liu, M. C., Kraus, A. L., Mann, A. W., \& Ireland, M. J. 2011, ApJ, 743, 148

[Cabrit et al.(2007)] Cabrit, S., Codella, C., Gueth, F., et al. 2007, A\&A, 468, L29

[Close et al.(2014)] Close, L. M., Follette, K. B., Males, J. R., et al. 2014, ApJl, 781, L30

[Cieza et al.(2017)] Cieza, L. A., Casassus, S., Pérez, S., et al. 2017, ApJ1, 851, L23

[Combet \& Ferreira(2008)] Combet, C., \& Ferreira, J. 2008, A\&A, 479, 481

[D’Orazi et al.(2018)] D’Orazi, V., Gratton, R., Desidera, S., et al. 2018, arXiv:1811.10621

[Dipierro et al.(2018)] Dipierro, G., Ricci, L., Pérez, L., et al. 2018, MNRAS, 475, 5296

[Dipierro et al.(2015)] Dipierro, G., Pinilla, P., Lodato, G., \& Testi, L. 2015, MNRAS, 451, 974

[Dong et al.(2015)] Dong, R., Zhu, Z., \& Whitney, B. 2015, ApJ, 809, 93

[Dong et al.(2016)] Dong, R., Zhu, Z., Fung, J., et al. 2016, ApJl, 816, L12

[Dong et al.(2018)] Dong, R., Liu, S.-y., Eisner, J., et al. 2018, ApJ, 860, 124

[Espaillat et al.(2014)] Espaillat, C., Muzerolle, J., Najita, J., et al. 2014, Protostars and Planets VI, 497

[Farinato et al.(2018)] Farinato, J., Bacciotti, F., Baffa, C., et al. 2018, arXiv:1808.00364

[Fedele et al.(2018)] Fedele, D., Tazzari, M., Booth, R., et al. 2018, A\&A, 610, A24

[Ferreira et al.(2006)] Ferreira, J., Dougados, C., \& Cabrit, S. 2006, A\&A, 453, 785

[Frank et al.(2014)] Frank, A., Ray, T. P., Cabrit, S., et al. 2014, Protostars and Planets VI, 451

[Garufi et al.(2016)] Garufi, A., Quanz, S. P., Schmid, H. M., et al. 2016, A\&A, 588, A8

[Guyon(2018)] Guyon, O. 2018, ARAA, 56, 315

[Hartmann \& Kenyon(1996)] Hartmann, L., \& Kenyon, S. J. 1996, ARAA, 34, 207

[Isella \& Turner(2018)] Isella, A., \& Turner, N. J. 2018, ApJ, 860, 27

[Kataoka et al.(2016)] Kataoka, A., Tsukagoshi, T., Momose, M., et al. 2016, ApJl, 831, L12

[Keppler et al.(2018)] Keppler, M., Benisty, M., Müller, A., et al. 2018, A\&A, 617, A44

[Li Causi et al.(2017)] Li Causi, G., Stangalini, M., Antoniucci, S., et al. 2017, ApJ, 849, 85

[Manara et al.(2014)] Manara, C. F., Testi, L., Natta, A., et al. 2014, A\&A, 568, A18

[Mattioli et al.(2018)] Mattioli, M., Pedichini, F., Antoniucci, S., et al. 2018, Ground-based and Airborne Instrumentation for Astronomy VII, 10702, 107024F

[Maurri et al.(2014)] Maurri, L., Bacciotti, F., Podio, L., et al. 2014, A\&A, 565, A110

[Mendigutía et al.(2018)] Mendigutía, I., Oudmaijer, R. D., Schneider, P. C., et al. 2018, A\&A, 618, L9

[Müller et al.(2018)] Müller, A., Keppler, M., Henning, T., et al. 2018, A\&A, 617, L2 
[Pedichini et al.(2017)] Pedichini, F., Stangalini, M., Ambrosino, F., et al. 2017, AJ, 154, 74

[Perrot et al.(2016)] Perrot, C., Boccaletti, A., Pantin, E., et al. 2016, A\&A, 590, L7

[Pollack et al.(1996)] Pollack, J. B., Hubickyj, O., Bodenheimer, P., et al. 1996, Icarus, 124, 62

[Schmid et al.(2018)] Schmid, H. M., Bazzon, A., Roelfsema, R., et al. 2018, A\&A, 619, A9

[Stamatellos \& Herczeg(2015)] Stamatellos, D., \& Herczeg, G. J. 2015, MNRAS, 449, 3432

[Stolker et al.(2017)] Stolker, T., Sitko, M., Lazareff, B., et al. 2017, ApJ, 849, 143

[Wagner et al.(2018)] Wagner, K., Follete, K. B., Close, L. M., et al. 2018, ApJ1, 863, L8

[Whelan et al.(2010)] Whelan, E. T., Dougados, C., Perrin, M. D., et al. 2010, ApJl, 720, L119

[Zhou et al.(2014)] Zhou, Y., Herczeg, G. J., Kraus, A. L., Metchev, S., \& Cruz, K. L. 2014, ApJl, 783, L17 